\title{
VALOR ACTUAL DE LA TOMOGRAFÍA POR EMISIÓN DE POSITRONES DURANTE EL SEGUIMIENTO DEL CÁNCER DE PRÓSTATA.
}

\author{
Aida Sánchez-Salmón y Álvaro Ruibal?.
}

Servicio de Medicina Nuclear. Complejo Hospitalario Universitario. Santiago de Compostela.

Profesor de Medicina Nuclearl. Facultad de Medicina. Universidad de Santiago. Santiago de Compostela. España.

\begin{abstract}
Resumen.- La Tomografía por Emisión de Positrones (PET) es una técnica de medicina nuclear, no invasiva, que utiliza radiofármacos y cámaras de detección de radiaciones algo diferentes a las empleadas en otras exploraciones isotópicas. La mayoría de las indicaciones son oncológicas, si bien, en nefrourología su uso ha sido menor debido a las características del radiofármaco hasta ahora más comúnmente usado, la 18F-FlúorDeoxiGlucosa (18F-FDG), un análogo de la glucosa que se elimina por vía renal, lo que dificulta su interpretación en las patologías urológicas tumorales. Un fármaco prometedor en este momento es la $18 \mathrm{~F}$ colina, tanto en estadificación como reestadificación,
\end{abstract}

Aida Sánchez-Salmón Medicina Nuclear Hospital Clínico Santiago de Compostela Av. Choupana, s/n 15706 la Coruña. (España) aida.sanchez.salmon@sergas.es especialmente si se usan equipos hibridos PET/TAC. La evolución de la PET en el carcinoma de próstata pasa por la utilización de otros radiofármacos que darán información sobre otros aspectos más específicos que modificarán las terapias y seguimiento del paciente.

Palabras clave: PET. Cáncer de próstata. Seguimiento.

Summary.- Positron emission tomography (PET) is a non-invasive nuclear medicine technology which uses radiotracers and cameras slightly different from the ones used in other nuclear medicine tests. Most current indications are for oncological diseases; in nephrology-urology its use has been reduced because of the characteristics of the most commonly used radiotracer, 18 F-fluor deoxiglucose (18F-FDG, a glucose analogue), which is excreted by the kidney, limiting the interpretation of the study in urologic malignancies. Currently, 18F-choline is a promising radiotracer for both staging and restaging, especially if hybrid PET/CT scan devices are used. New radiotracers will be needed in the evolution of PET to obtain information about more specific aspects of prostate carcinoma that will modify therapy and follow-up.

Keywords: PET. Prostate cancer. Follow-up. 


\section{INTRODUCCIÓN}

La Tomografía por Emisión de Positrones (PET) es una técnica de reciente incorporación a la medicina nuclear, no invasiva, que utiliza radiofármacos y cámaras de detección de radiaciones algo diferentes a las empleadas en otras exploraciones isotópicas. Los radiofármacos contienen radionúclidos (isótopos radiactivos) emisores de positrones, de alta energía y una vida media muy corta, por lo que es necesaria una cámara de detección especial (PET) que puede o no llevar asociado un TAC. Lo importante de esta técnica es que ofrece una información funcional (bioquímico-metabólica) de alta sensibilidad, lo que la diferencia de otras exploraciones de diagnóstico por la imagen que hacen hincapié preferentemente en los aspectos morfológicos. Hasta ahora su uso clínico era tutelado y sólo estaban autorizadas unas indicaciones concretas, principalmente oncológicas. Ahora, tras el Informe $\mathrm{n}^{\circ} \mathbf{4 6}$ de la Agencia de Evaluación de Tecnologías Sanitarias, las cosas pueden cambiar notablemente, pues los resultados obtenidos a lo largo de estos años han demostrado realmente su utilidad práctica en oncología. Quizás merezca destacarse que el $88 \%$ de los médicos la consideraron una exploración útil, que evitó pruebas invasivas y con riesgo en el $76 \%$ de los pacientes y que obvió terapias innecesarias en un porcentaje similar. En nefrourología su uso ha sido menor debido a las características, especialmente farmacodinámicas, del radiofármaco hasta ahora más comúnmente usado, la 18F-FlúorDeoxiGlucosa (18F-FDG), un análogo de la glucosa que se elimina por vía renal. Asimismo, en el caso concreto del carcinoma de próstata, la actividad radiactiva retenida en la vejiga ha limitado notablemente los estudios PET.

Sin embargo, la evolución continua de esta técnica, tanto a nivel de instrumental cada vez más precisa lequipos combinados de TAC y PET, y próximamente, de RM y PET), como del desarrollo de nuevos radiofármacos más específicos, permite ser muy optimista y creer que asistiremos, muy probablemente, a una revolución en esta modalidad de imagen (1) y su empleo en la clínica diaria.

\section{RADIONUCLIDOS EMISORES DE POSITRONES}

Los isótopos radiactivos emisores de positrones fueron descubiertos en los años 30 del siglo pasado $y$, en su gran mayoría, son isótopos de elementos biológicos presentes en cualquier molécula del organismo: carbono (11C, vida media: 20.4 minutos), oxígeno (150, vida media: 2.1 minutos) y nitrógeno (13N, vida media: 9.96 minutos) y, como no hay ningún hidrógeno emisor de positrones, se utiliza el flúor (18F, vida media:109,8 minutos) en su lugar. Ello hace que cualquier molécula del organismo pueda ser, en teoría, marcada con un isótopo radiactivo y utilizada para evidenciar un hecho bioquímico-molecular. Existen otros isótopos emisores de positrones no biológicos como el rubidio (82Rb) y el galio $(68 \mathrm{Ga})$. Conviene recordar que los isótopos emisores de positrones tienen una actividad específica elevada y no alteran las características fisiológicas de las moléculas, lo cual, unido a su cortísima vida media (tiempo en el que emite la radiación), permiten obtener imágenes de alta calidad con baja exposición radiactiva para el paciente y con sensibilidades del orden de picomoles.

\section{UTILIDAD DE LA 18F-FDG}

El único radiofármaco autorizado para uso clínico en nuestro país es la 18F-FDG (fluor-2-deoxiglucosa, marcada con 18 F) que tiene una vida media de 109,8 minutos. La 18F-FDG es captada por las células del organismo de un modo similar al de la glucosa; luego es fosforilada por la hexoquinasa, pero, a diferencia de aquella, no experimenta más pasos metabólicos, pues no es un sustrato adecuado para la fosfoglucosa isomerasa ya que carece del $-\mathrm{OH}$ en posición $\mathrm{C} 2$; en consecuencia queda atrapada, por lo que su "acúmulo" es un indicador del consumo de glucosa por parte de las células (Figura 1). Los tejidos tumorales presentan una glucolísis acelerada y una menor capacidad para generar energía vía la glucolísis aeróbica, por lo que la captación de glucosa se incrementa notablemente. De ahí que sea precisamente la oncología una de las aplicaciones clínicas más importantes de la PET. Sin embargo, hay que tener en cuenta que, dado que existe un consumo de glucosa por todas las células del organismo, incluso en condiciones fisiológicas, no podemos establecer que todo acúmulo de 18F-FDG sea sinónimo de tumor, pues tejidos en reparación, inflamaciones y otros procesos no neoplásicos pueden determinar un incremento tisular de glucosa. Por ello, la PET con 18F-FDG resulta una exploración muy sensible, pero poco específica. Asimismo, todos aquellos factores que condicionen un reducido consumo de glucosa por parte de una lesión tumoral harán que no sea detectable por esta técnica de imagen, dando lugar a un falso negativo. Sin embargo, y a pesar de estos inconvenientes, el uso de 18F-FDG ha permitido avanzar en la estadificación y control de muchos tumores, modificando en un alto porcentaje de casos la planificación terapéutica y aportando una información pronóstica de interés práctico.

\section{Diagnóstico inicial con 18F-FDG}

El cáncer de próstata muestra una amplia actividad biológica que va desde los tumores de bajo grado, indolentes, a tumores muy agresivos con ele- 
vada tendencia a la diseminación. Por ello, la posible utilidad de la PET va a depender las características biológicas de la neoformación maligna. Como ocurre en otros tumores, el estadío inicial es muy importante en pacientes con carcinoma de próstata, pues ayuda a elegir el tratamiento adecuado y valorar el pronóstico. La extensión extracapsular, la invasión de las vesículas seminales o de ganglios serán condicionantes de la evolución ulterior.

Los estudios de imagen deben ser seleccionados de forma individualizada, teniendo en cuenta la estratificación de riesgo, en la que se combinan la concentración del antígeno prostático específico (PSA), la escala de Gleason, o el estadío clínico. La técnica más comúnmente utilizada es la ecografía transrectal, aunque presenta algunas limitaciones y no es adecuada para valorar la afectación ganglionar o a distancia. El uso de la tomografía axial computarizada (TAC) es limitado dado que carece

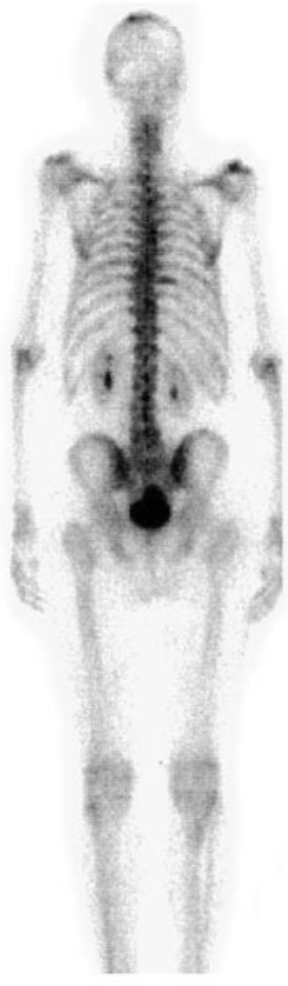

Gammagrafía ósea

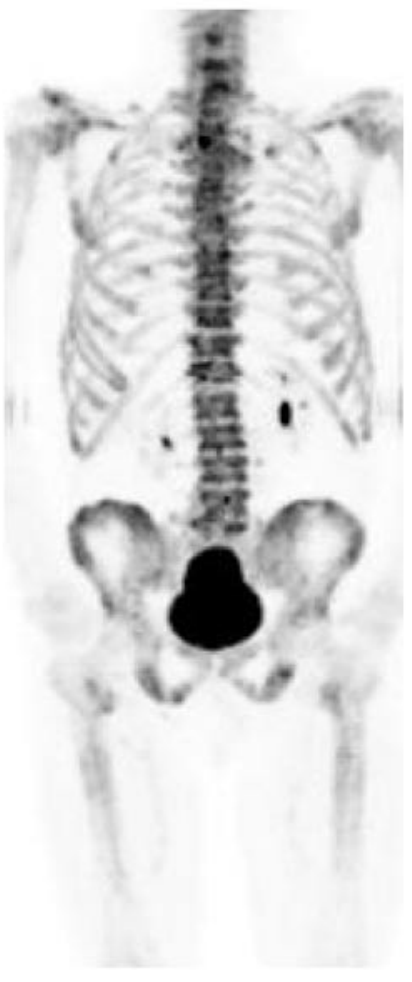

${ }^{18}$ F-fluoride de resolución en tejidos blandos, aunque se emplea en la planificación de la radioterapia y braquiterapia. La resonancia nuclear magnética (RNM) parece ser la exploración más exacta ya que, aunque no es perfecta, puede definir el tumor primitivo, la invasión extracapsular potencial y/o la perineural. También es posible el uso de espectroscopia con RM gracias a la asociación de alteraciones en la concentración de ciertos metabolitos como el aumento de colina o la disminución de citrato en la próstata. Sin embargo, todas estas técnicas dependen, a menudo, de la experiencia del especialista, y a veces, no es posible usarlas en todos los pacientes.

En relación con las pruebas de imagen metabólicas, la PET con 18F-FDG resulta de utilidad en tumores con alta actividad glucolítica lasociada a cambios cuali y cuantitativos en la expresión de algunos enzimas y transportadores de glucosa), elevado crecimiento y desdiferenciación celular. Algunos de estos procesos se han analizado en cultivos celulares de cáncer de próstata $y$, aunque no se ha demostrado una relación clara entre estas alteraciones bioquímicas y la captación de 18F-FDG, parece que ésta puede detectar de forma más selectiva los tumores más agresivos que dependan del metabolismo
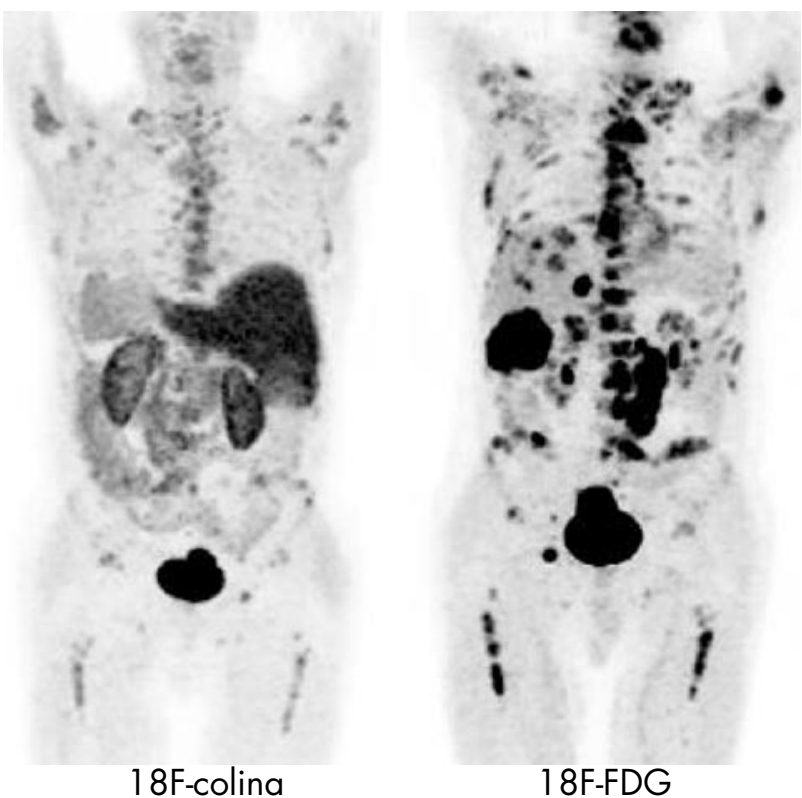

FIGURA 1. Paciente con adenocarcinoma de próstata, estadío T3b-4, N 1, que en el seguimiento presenta niveles de PSA en ascenso, tratado con Hormono y Radioterapia.

En la gammagrafía ósea, se observa una zona sospechosa de malignidad en el noveno arco costal, radiografía negativa.

En la exploración con ${ }^{18} \mathrm{~F}$-fluoride: Múltiples lesiones óseas. El estudio con ${ }^{18} \mathrm{~F}$-colina ofrece resultados similares, con metabolismo algo disminuído debido al tratamiento hormonal. Con ${ }^{18}$ F-FDG se observan lesiones óseas, hepáticas

y múltiples lesiones ganglionares en mediastino y retroperitoneo.

(Cortesía del Dr Werner Langsteger, St Vincent's Hospital-Linz Austria). 
de glucosa. De hecho se ha demostrado in vitro que la captación de 18F-FDG es superior en los cánceres con mayor puntuación en la escala de Gleason. También algunos estudios clínicos han descrito que la captación de 18-FDG se correlaciona con la concentración sérica y la velocidad de PSA. Sin embargo, no todos los autores llegan a las mismas conclusiones. Jadear y cols. (2) demuestran en estudios experimentales que el metabolismo glucídico fue mayor en las líneas independientes de andrógenos (PC-3) que en las sensibles (CWR-22), por lo que la 18F-FDG podría ser de utilidad para evaluar la respuesta a la terapia ablativa androgénica y conocer precozmente la falta de respuesta en pacientes con cáncer de próstata metastático.

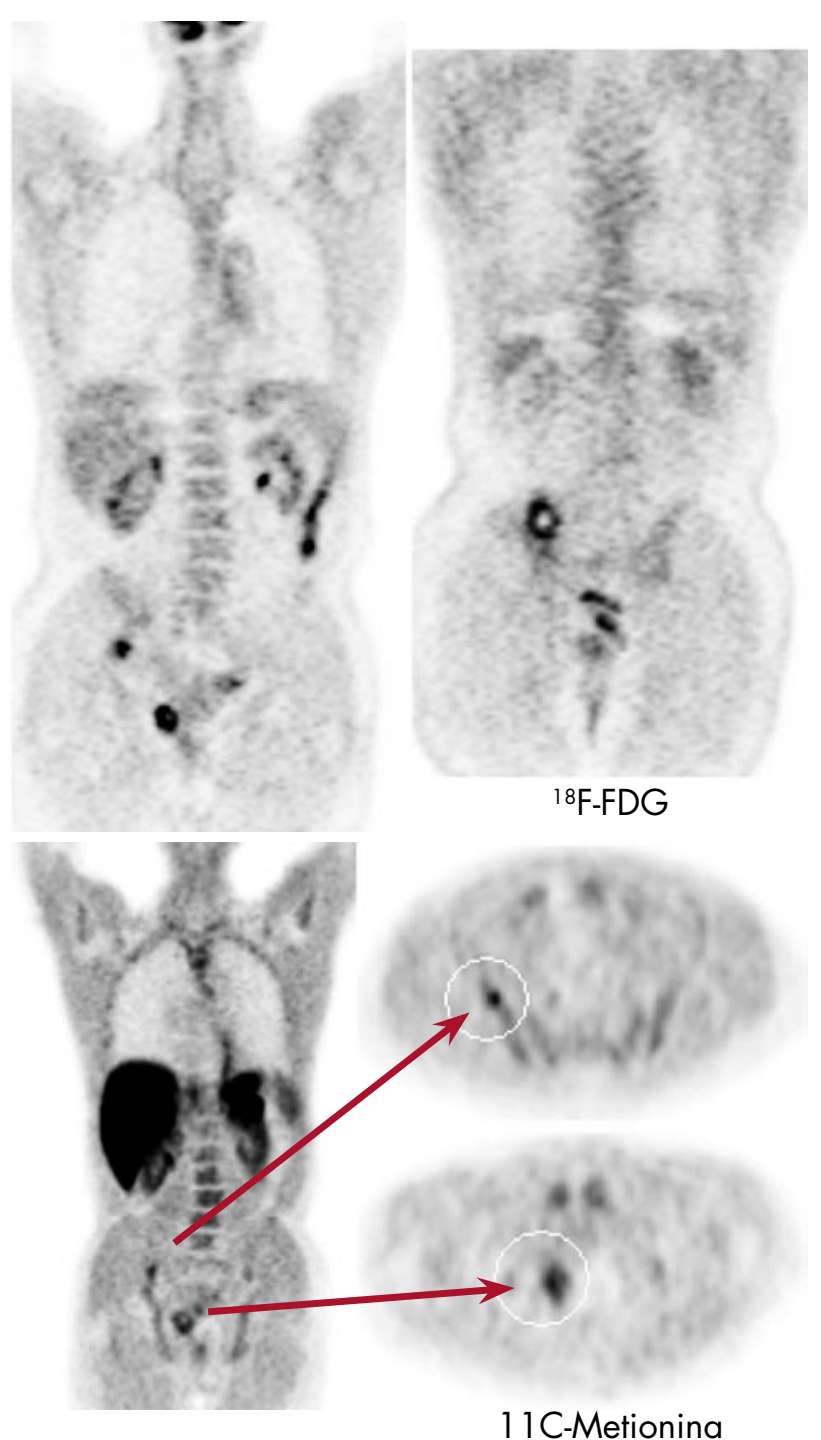

Los primeros estudios PET fueron descorazonadores, probablemente por la suma de varias causas, entre las que figuran la excreción urinaria de 18F-FDG, la mala selección de pacientes o los algoritmos antiguos de reconstrucción de imágenes. A diferencia de lo que ocurre en otros tumores, la mayoría (hasta en el $81 \%$ de los caso en algunos estudios) de los carcinomas de próstata muestran una relativa baja captación de FDG, pudiendo ser consecuencia de un lento crecimiento y/o un escaso tamaño (3) Se han realizado esfuerzos para reducir el artefacto producido por la excreción de orina, ya sea por medio de métodos de irrigación continua o forzando la diuresis, pero ello no se ha acompañado de mejores resultados. Otro problema añadido es la similitud en la captación de 18F-FDG entre tumores e hipertrofias prostáticas benignas (4-5). Podemos decir que la sensibilidad de la PET sigue siendo baja (alrededor del $60 \%$ ) y lo que hace que no se pueda recomendar como técnica de uso generalizado en la evaluación inicial de estos pacientes (6).

Desde el punto de vista diagnóstico, es de gran valor conocer si el tumor está confinado en la glándula o bien existe diseminación local, regional o a distancia, pues ello incide directamente en el pronóstico y evolución posterior. La escala de Gleason y la valoración del PSA pueden ser de ayuda, mientras
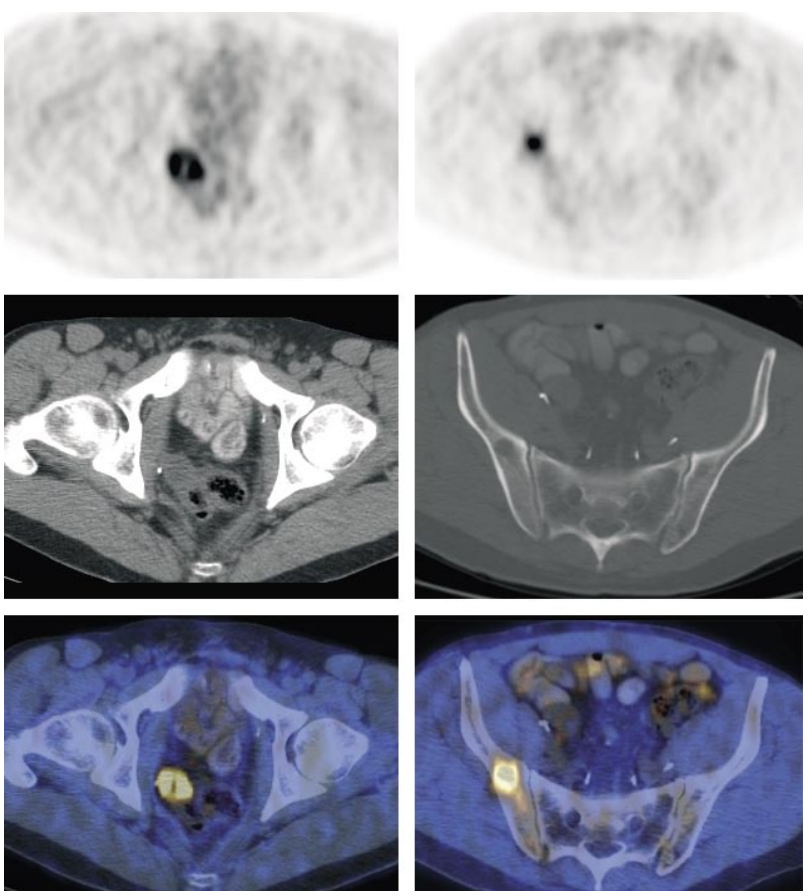

${ }^{18}$ F-FDG PET/TAC

FIGURA 2. Paciente en seguimiento por carcinoma de próstata estudiado con PET/TAC con ${ }^{18 F-F D G ~ y ~} 11$ C-metionina. Con ambos radiofármacos, se observa una lesión ósea en la cresta iliaca y de un ganglio perirrectal.

(Cortesía de los Dres, H. Schoder y T. Akhurst del Memorial Sloan Kettering Cancer Center, NY, Estados Unidos). 
que las técnicas morfológicas de imagen se basan en criterios dependientes del tamaño, careciendo de especificidad y sensibilidad. El uso de la gammagrafía ósea tampoco es de gran valor, dada la escasa probabilidad de encontrar metástasis óseas en el momento del diagnostico, sobre todo, cuando la concentración sérica del PSA es reducida.

La utilidad de la PET con 18F-FDG tampoco es lo suficientemente buena como para recomendar el uso rutinario de esta exploración, ya que la evaluación de ganglios se ve severamente afectada por la eliminación normal del radiofármaco por la orina, su depósito en la vejiga (7) y por la escasa actividad metabólica de las lesiones. Por tanto, tan sólo se podría recomendar en los tumores de alto grado, con la finalidad de reducir el porcentaje de infraestadificaciones que puede ocurrir en el $30-60 \%$ de los pacientes que se operan por enfermedad clínicamente localizada.

\section{Seguimiento y sospecha de recidivas o metástasis con 18F-FDG}

En la mayoría de los casos la recurrencia se presenta como una alteración bioquímica, que se refleja en la elevación del PSA. En muchas ocasiones esta elevación no se acompaña de otros síntomas clínicos siendo los estudios de imagen frecuentemente negativos.

La PET con 18F-FDG parece ser algo más sensible que el TAC para la detección de metástasis ganglionares, si bien, en varios estudios se ha establecido una cifra de PSA a partir de la cual los resultados son mejores. Cuando el PSA es mayor de $4 \mathrm{ng} / \mathrm{ml}$, la sensibilidad de la PET es del $35-50 \%$ y su valor predictivo positivo es elevado, con escasos casos de falsos positivos, perdiéndose este valor si las cifras de PSA son menores de $2.4 \mathrm{ng} / \mathrm{ml}$ tras prostatectomía. $(6,8-9)$. Conviene recordar que el tamaño tumoral y su biología van a ser factores condicionantes de la positividad de la PET en los pacientes en seguimiento.

Donde parece tener más valor es en la detección de metástasis a distancia (10). En el caso de las metástasis óseas, los primeros estudios publicados demostraban una alta sensibilidad y especificidad de la PET, superiores, para algunos grupos, a las de la gammagrafía ósea (1 1-12). En la actualidad parece claro que las diferencias en la sensibilidad de la PET y la gammagrafía ósea, son debidas a la visualización de diferentes aspectos de la lesión ósea: la 18FFDG se acumula con mayor intensidad en las lesiones líticas que en las escleróticas, por lo que parece menos útil para la detección de éstas en pacientes no tratados, pero, sobre todo en los que han sido tratados previamente (13). Otro aspecto de interés es la posibilidad de diferenciar lesiones óseas activas y quiescentes (14). Un motivo potencial para la utilización de 18F-FDG en lugar de gammagrafía ósea se da cuando se quiere evitar el fenómeno de captación "en llamarada" (8) que ocurre en la gammagrafía ósea tras el tratamiento y puede persistir varios meses, de forma que sería posible diferenciar progresión de este fenómeno (15). En pacientes sometidos a quimioterapia que actúa sobre los microtúbulos, se ha podido ver que la $18 \mathrm{~F}-\mathrm{FDG}$ fue de gran utilidad junto al PSA; así, cuando el aumento del PSA fue $>25 \%$, la PET identificó correctamente el estadio clínico del paciente en más del $90 \%$ de los pacientes. También incrementos $>33 \%$ en la SUV (Standardized uptake value) pueden ayudar a definir pacientes que no responden al tratamiento (16).

\section{OTROS RADIOFÁRMACOS}

Dadas las limitaciones de la 18F-FDG en la evaluación de los carcinomas prostáticos y un mejor conocimiento de la biología de estos tumores, se han desarrollado y obtenido otros radiofármacos, algunos de los cuales pueden ser de interés práctico. Podemos destacar los siguientes:

\section{C- acetato}

Se trata de un radiofármaco utilizado en otras indicaciones oncológicas y cardiológicas (metabolismo beta oxidativo). Dado que su excreción no se efectúa por vía urinaria, se consideró la posibilidad de emplearlo en la evaluación de los tumores prostáticos. La captación celular de acetato se relaciona con la síntesis de lípidos y no es específica de tumor, mostrando valores similares en carcinomas de próstata, hombres mayores de 50 años con próstata normal y pacientes con hipertrofia prostática benigna (6). No está clara su relación con la concentración de PSA. Otro problema que presenta este radiofármaco es su disponibilidad, pues la vida media o período de semidesintegración es de 20 minutos, por lo que es obligada la presencia de un ciclotrón próximo al PET para su utilización. Se está intentando marcar el acetato con $18 \mathrm{~F}$, lo que permitiría ampliar las series de pacientes estudiados, hasta ahora muy escasas y precisar mejor sus posibles indicaciones. (17) $\mathrm{A}$ pesar de todo lo anterior, este radiofármaco se ha mostrado, para algunos grupos, más útil que la FDG en la detección de metástasis en tejidos blandos y ganglios linfáticos (8).

\section{C-colina/ 18 F-colina}

Tras haberse demostrado por otros métodos, como la espectroscopia con RNM, que el carcinoma de próstata cursaba con una mayor concentración de 
colina, se iniciaron estudios encaminados a conocer su posible interés práctico. Existen varios compuestos de colina, algunos marcados con $11 \mathrm{C}$ y otros con 18F. Si bien presentan algunas características farmacodinámicas distintas entre ellos, todos sufren un rápido aclaramiento sanguíneo y una precoz captación por la próstata.

Aunque parece ser que la 18 F-colina ofrece una ligera mejor calidad de la imagen en relación a la 11 C-colina, debemos recordar que los compuestos marcados con $18 \mathrm{~F}$ se eliminan por la orina, lo que conlleva su acumulo en la vejiga y una dificultad adicional para la valoración de las imágenes. Además, este radiofármaco tampoco es específico de tumor, por lo que es posible ver captaciones aumentadas en las hipertrofias prostática benignas, granulocitos, macrófagos y ganglios linfáticos reactivos. Breeuwsma y cols. (18) han observado que la captación de 11C-colina no se correlaciona con la proliferación celular en el cáncer prostático humano, lo que induce a pensar en la existencia de otros factores que influyen en su captación tisular. Por todo ello, no se aconseja en la estadificación inicial del carcinoma prostático (19). Sin embargo, otro estudio reciente (20) ha demostrado una sensibilidad del 100\% para los tumores primitivos, superiores a las de la RM (60\%) y espectroscopia-RM (65\%). La captación del radiofármaco se correlacionó estadísticamente con los valores del PSA sérico $(r=0.52)$ y los resultados de la PET concordaron con los de la anatomía patológica en el $81 \%$ de los casos y superaron a los de espectroscopia (50\%). A pesar de realizarse con 20 pacientes, los resultados obtenidos son muy buenos y superiores a los de la 18F-FDG en la detección de lesiones ganglionares y óseas.(21-24). Se han descrito captaciones del radiofármaco en un $35 \%$ de las neoplasias prostáticas intraepiteliales y en los carcinomas esta exploración presentó una sensibilidad, especificidad, eficacia, VPP y VPN del $66 \%, 81 \%$, $71 \%, 87 \%$ y $55 \%$ respectivamente. Merece destacarse el alto número de falsos negativos. Por todo ello, no se recomienda su uso rutinario en los sujetos de alto riesgo (25). Este mismo solapamiento de la captación del radiofármaco entre tumores e hiperplasias ha sido descrito por otros autores (26).

El uso de colina como marcador, aunque en series pequeñas, está ofreciendo resultados muy prometedores en la reestadificación del cáncer de próstata. Recientemente, aunque con un escaso volumen de pacientes, Schmid y cols. (26) usaron 18 F-colina y equipo PET/TAC en la sospecha de recurrencia bioquímica, incluso con cifras de PSA bajas $(<5 \mathrm{ng} / \mathrm{ml})$, con hallazgos tanto en el lecho prostático como a nivel ganglionar local y óseo. También se observó captación por parte de ganglios mediastínicos en pa- cientes con sarcoidosis, y en otras patologías no relacionadas como, por ejemplo, el meningioma. Parte de estos buenos resultados se deben al uso de una equipo híbrido PET /TAC que, al incorporar ambas modalidades de imagen reducen los resultados falsos positivos. Langsteger y cols. (23) también han descrito excelentes resultados en el seguimiento de carcinomas de próstata con elevaciones de PSA, por lo que se ha recomendado la realización de 18 F-colina PET/TC sin restricción en la elevación del PSA (27). También otros grupos aconsejan su utilización en la reestadificación de recidivas y en la monitorización de los pacientes con cáncer de próstata (19).

Para obviar las captaciones del radiofármaco por parte de procesos no tumorales, se están incorporando cambios en la dinámica de las exploraciones y actualmente, ya se están incorporando otras metodologías, como la realización de imágenes dinámicas o la exploración en dos fases, que permite la diferenciación entre tejidos de naturaleza benigna y maligna, en función del comportamiento de la captación del trazador. Así, Kwee y cols. (28) realizan una detección a los 7 minutos y 1 hora de la inyección del radiofármaco y observan que las lesiones malignas cursan con una captación que se incrementa con el tiempo (mediana 14\%), mientras que en las no malignas aquella desciende (mediana de la reducción $17 \%$ ). Por ello recomiendan su uso rutinario.

Algunos centros en Europa han incorporado la PET con 18F-colina en el diagnóstico inicial en un grupo seleccionado de pacientes de alto riesgo, en función de la escala de Gleason, la cifra de PSA o el tiempo de duplicación de PSA. Todo ello ha conllevado cambios en el estadío, generalmente para elevarlo, de muchos pacientes lo que se ha traducido en la actitud terapéutica (23).

Como en el caso de la 18F-FDG, la captación de $18 \mathrm{~F}$-colina puede verse reducida en las metástasis durante el tratamiento hormonal (antiandrógenos) del cáncer de próstata. Sin embargo aún no se sabe si esta disminución es paralela a la de las cifras de PSA.

\section{F-Fluor}

El ión 18F-Fluor, es un radiofármaco descrito en los años 60 del siglo pasado y usado en la imagen ósea. Su extracción de la sangre es mucho más alta y rápida que la de otros agentes usados como los fosfonatos. Sin embargo, su uso es mucho menos frecuente y no de rutina por su escasa disponibilidad. A pesar de ello, es preciso destacar la extremadamente alta calidad de la imagen ósea que se obtiene con el uso de este ión, con un contraste muy elevado 
entre la captación por parte de la lesión tumoral y el hueso normal. Otra ventaja de este radiofármaco es la posibilidad de detección temprana de metástasis óseas, tanto líticas como blásticas (8). Una posible limitación radica en que, debido a su altísima sensibilidad, es posible la detección de cambios degenerativos mínimos. Para obviar los posibles falsos positivos es interesante realizar la exploración con equipos híbridos PET/TAC (23).

En un reciente estudio comparativo entre gammagrafía ósea con SPECT (tomagráfica) y la PET/ TAC con ión 18F-Fluor y realizado en pacientes con carcinoma de próstata de alto riesgo, se estableció la superior especificidad y sensibilidad de la PET/TAC, más específico que la realización de sólo PET con ión 18F-Fluor y más sensible y específico que la gammagrafía con SPECT (29). Las sensibilidades, especificidades, valores predictivos positivos y negativos fueron los siguientes: gammagrafía ósea planar 70 , $57,64$ y $85 \%)$, gammagrafía ósea con SPECT (82, $82,86$ y $90 \%), 18 \mathrm{~F}(100,62,74$ y $100 \%)$ y $18 \mathrm{~F}$ PET/TAC (100\% todas) (29). Queda por precisar la comparación en cuanto a coste-efectividad entre la gammagrafía ósea y el ión 18F-Fluor, pues su indicación clínica es la misma para ambas exploraciones.

\section{C-metionina}

Todavía se desconoce el mecanismo exacto de captación de este aminoácido por los tumores, aunque parece estar en relación con su transporte celular y la síntesis de proteínas. Debe tenerse presente que el destino del aminoácido marcado y la eliminación de los metabolitos radiactivos inciden notablemente en el análisis cuantitativo del radiofármaco. La escasa eliminación por vía urinaria de este trazador, debido a su mayor metabolización en páncreas e hígado, ha permitido también su uso en tumores no cerebrales, como pélvicos, especialmente de vejiga, y ginecológicos, aunque debido a la captación normal intestinal, su evaluación puede ser muy difícil (1). Toth y cols. (30) describen en los pacientes con sospecha de carcinoma de próstata una positividad del $75 \%$, comprobándose en el $47 \%$ un cáncer tras la realización de una biopsia, por lo que la aconsejan en pacientes con altas concentraciones séricas de PSA y biopsias negativas de forma repetida.

En estudios comparativos entre la captación de 11 C-metionina y 18F-FDG, se ha observado un acumulo celular más rápido con la metionina, siendo superior a ésta en la detección de tumor primario y lesiones metastásicas.

\section{F-Fluorodihydrotestosterona (FDHT)}

La presencia de receptores de andrógenos juega un papel importante en la proliferación y crecimien- to del carcinoma prostático. La posibilidad de objetivar su expresión y ocupación por ligandos, significaría un gran paso para el control terapéutico (31). Los estudios con este radiofármaco están en una fase muy inicial, pero cabe pensar, que la captación de FDG por una lesión prostática que no capte FDHT puede caracterizar a un subgrupo de pacientes con peor pronóstico, ya que sería sugestivo de andrógeno-independencia. $(8,32$.). Dehdashti et al (33) obtienen en pacientes con carcinoma de próstata diseminado unos buenos resultados, evidenciando el $86 \%$ de las lesiones conocidas y un porcentaje muy alto de lesiones no sospechadas. Es interesante resaltar que los casos PET positivos se correlacionaron con altas concentraciones de PSA sérico $y$, posiblemente, con un mayor volumen tumoral.

\section{¿CUÁl ES EL FUTURO?}

El futuro de la PET en carcinoma de próstata, como en otros tumores, pasa por la utilización de moléculas marcadas que exploren diferentes aspectos de la enfermedad como la hormonodependencia, agresividad biológica, sensibilidad a determinados tratamientos o la respuesta terapéutica. He aquí algunos ejemplos de nuevas moléculas y su utilidad.

18F- hidroxiflutamida: Un alto porcentaje de los carcinomas prostáticos son andrógeno-dependientes en el momento del diagnóstico, Por ello, el uso de antiandrógenos marcados puede ser una interesante línea de investigación con la finalidad de evidenciar dichas neoformaciones. Uno de ellos es la hidroxiflutamida, derivado de la flutamida, que se ha mostrado de utilidad en estudios experimentales (34).

Ligandos para el antígeno prostático específico de membrana: El PSMA está sobreexpresado en el cáncer de próstata, incluso en los hormonoindependientes. Se han descrito pequeñas moléculas capaces de unirse a este antígeno y marcadas con $11 \mathrm{C}$ que permiten su visualización. (35).

Bombesina: Su receptor está sobreexpresado en muchos tumores, incluído el prostático. Se han desarrollado radiofármacos (18F-FB-lisina (3) bombesina) capaces de evidenciar estos receptores presentes en tumores prostáticos experimentales (36).

Control de terapia génica: Mullerad y cols. (37) han demostrado cambios en la intensidad del metabolismo de 18F-FDG tras el tratamiento génico oncolítico con virus herpes simple en animales y concluyen que la PET con FDG puede se un buen método de selección de los pacientes que pueden beneficiarse de esta técnica. Similares conclusiones obtienen Johnson y cols (38). También, recientemente, se han 
descrito otras estrategias genéticas en cánceres prostáticos recurrentes que expresan receptor de andrógenos y PSA (39).

Otro aspecto que mejorará en el futuro es la disponibilidad de equipos PET en nuestro país. La evolución del número de instalaciones PET en España ha sido muy rápida en los últimos años, tanto en la sanidad privada como en la pública, si bien, es preciso recordar que, en este momento, la indicaciones de próstata no están admitidas en el Sistema Nacional de Salud de forma rutinaria y es precisa la valoración de cada caso por diferentes comisiones que aceptarán la exploración PET, especialmente si dependiendo de sus resultados se adoptarán medidas terapéuticas diferentes.

También los equipos están evolucionando, incorporándose los híbridos PET/TC, que aunque, con algunas limitaciones, aportan el gran beneficio de incorporar la información del metabolismo (PET) a la morfología (TAC) y acortan el tiempo de exploración al paciente (40).

\section{CONCLUSIONES}

En el momento actual, el fármaco más comúnmente empleado en PET, la 18F- FDG, tiene una utilidad restringida en el cáncer de próstata. Parece ser de utilidad en carcinomas agresivos y hormonaresistentes, así como en la valoración de metástasis óseas de determinados casos. El fármaco más prometedor es en este momento la 18F-colina, tanto en estadificación como reestadificación, especialmente si se usan equipos híbridos PET/TAC que aúnan la imagen metabólica con la morfológica, aportando una información más completa. Sin embargo, creemos que la evolución de la PET en el carcinoma de próstata pasa también por la utilización de otros radiofármacos que darán información sobre otros aspectos más específicos, concretos y valiosos a la hora de diagnosticar y seguir al paciente.

\section{BIBLIOGRAFÍA y LECTURAS RECOMENDADAS ( ${ }^{*}$ lectura de interés $y^{* *}$ lectura fundamental)}

1. COOK, G.J.R.; MAISEY, M.N.; FOGELMAN, I.: "Normal Variants, artefacts, and interpretative pitfalls in PET imaging with 18-fluoro-2-glucose and carbon-11 methionine". Eur. J. Nucl. Med., 26: $1363,1999$.
2. JADEAR, S.; XIANKLUI, L.; SAINAN, A. y cols.: "Glucosa metabolism of human prostate cancer Mouse xenografts". Mol. Imaging., 4: 91, 2005.

3. EFFERT, P.J.; BARES, R.; HANDT, S. y cols.: "Metabolic imaging of untreated prostate cancer by positron emission tomography with 18 fluorinelabeled deoxyglucose". J. Urol., 155: 994, 1996.

4. LUI, I.J.; ZAFAR, M.B.; LAI, Y.H. y cols.: "Fluorodeoxyglucose positron emission tomography studies in diagnosis and staging of clinically organ confined prostate cancer". Urology, 57: 108, 2001.

5. OYAMA, N.; AKINO, H.; SUZUKI, Y. y cols.: "The increased accumulation of (18F)fluorodeoxyglucose in untreated prostate cancer". Jpn. J. Clin. Oncol., 29: 623, 1999.

6. SCHÖDER, H.; LARSON, S.M.: "Positron Emission Tomography for prostate, bladder and renal cancer". Semin. Nucl. Med., 34: 274, 2004.

7. EL-HADDAD, G.; ALAVI, A.; MAVI, A. y cols.: "Normal variants in (18F)-fluorodeoxyglucose PET imaging”. Radiol. Clin. N. Am., 42: 1063, 2004.

**8. JANA, S.; BLAUFOX, M.D.: "Nuclear medicine studies for prostate, testes, and bladder". Semin. Nucl. Med., 36: 51, 2006.

9. SCHODER, H.; HERRMANN, K.; GONEN, M. y cols.: "1-18F-fluoro-2deoxyglucose positron emission tomography for the detection of disease in patients with prostate-specific antigen relapse after radical prostatectomy". Clin. Cancer. Res., 11: 4761, 2005.

10. KUMAR, R.; ZHUANG, H.; ALAVI, A.: "PET in the management of urologic malignancies". Radiol. Clin. N. Am., 42: 1141, 2004.

11. NÚÑEZ, R.; MACAPINLAC, H.A.; YEUNG, H.W. y cols.: "Combined 18F-FDG and 11C-methionine PET scans in patients with newly progressive metastatic prostate cancer". J. Nucl. Med., 43: 46, 2002.

12. KAO, C.H.; HSIEH, J.F.; TSAI, S.C. y cols.: "Comparison and discrepancy of 18F-2-deoxyglucose positron emission tomography and Tc- $99 \mathrm{~m}$ MDP bone scans to detect bone metastases". Anticancer Res., 20: 2189, 2000.

13. SHREVE, P.D.; GROSSMAN, H.B.; GROSS, M.D. y cols.: "Metastatic prostate cancer: Initial findings of PET with 2-deoxy-2 (F-18) Fluoro-Dglucose". Radiology, 199: 751, 1996.

*14. MORRIS, M.J.; AKHURST, T.; OSMAN, I. y cols.: "Fluorinated deoxy glucose positron emission tomography imaging in progressive metastatic prostate cancer". Urology, 59: 913, 2002.

15. GARCÍA, J.R.; SIMÓ, M.; SOLER, M. y cols.: "Relative roles of bone scintigraphy and positron emission tomography in assessing the treatment response of bone metastases". Eur. J. Nucl. Med. Mol. Imaging., 32: 1243, 2005. 
16. MORRIS, M.J.; AKHURST, T.; LARSON, S.M. y cols.: "Fluorodeoxyglucose positron emisión tomography as an outcome measure for castrate metastatic prostate cancer treated with antimicrotubule chemotherapy". Clin. Cancer Res., 11: 3210, 2005.

17. PEÑUELAS SÁNCHEZ, I.: "Radiofármacos PET". Rev. Esp. Med. Nuclear, 20: 477, 2001.

18. BREEUWSMA, J.A.; PRUIM, J.; JONGEN, M.M. y cols.: "In vivo uptake of $11 \mathrm{C}$-choline does not correlate with cell proliferation in human prostate cancer". Eur. J. Nucl. Med. Mol. Imaging., 32: 668, 2005.

19. YOSHIDA, S.; NAKAGOMI, K.; GOTO, S. y cols.: "11C-choline positron emission tomography in prostate cancer: primary staging and recurrent site staging". Urol. Int., 74: 214, 2005.

20. YAMAGUCHI, T.; LEE, J.; UEMURA, H. y cols.: "Prostate cancer: a comparative study of 11C-choline PET and MR imaging combined with proton MR spectroscopy". Eur. J. Nucl. Med. Mol. Imaging., 32: 742, 2005.

21. DE JONG, I.J.; PRUIM, J.; ELSINGA, P.H. y cols: "Visualization of prostate cancer with 11Ccholine positron emission tomography". Eur. Urol., 42: 18, 2002.

22. DE JONG, I.J.; PRUIM, J.; ELSINGA, P.H. y cols: "Preoperative staging of pelvic lymph nodes in prostate cancer by 11C-choline PET". J. Nucl. Med., 44: 331, 2003.

**23. LANGSTEGER, W.; HEINISCH, M.; FOGELMAN, I.: "The role of fluorodeoxiglucose, 18F-Dihydrophenilalanine, 18F-Choline, and 18F-Fluoride in bone imaging with emphasis on prostate and breast". Semin. Nucl. Med., 36: 73, 2006.

24. KWEE, S.A.; COEL, M.N.; LIM, J. y cols.: "Prostate cancer localization with 18fluorine-Fluorocholine positron emission tomography". J. Urol., 173: 252, 2005.

25. FARSAD, M.; SCHIAVINA, R.; CASTELLUCCI, P. y cols.: "Detection and localization of prostate cancer: correlation of 11C-Choline PET/CT with histopathologic step-section analysis". J. Nucl. Med., 46: 1642, 2005.

26. SCHMID, D.T.; JOHN, H.; ZWEIFEL, R. y cols.: "Fluorocholine PET/CT in patients with prostate cancer: initial experience". Radiology, 235: 623, 2005.

*27. HEINISCH, M.; DIRISAMER, A.; LOIDL, W. y cols.: "Positron Emisión Tomography/ computed tomography with F-18-fluorocholine for restaging of prostate cancer patients: meaningful at PSA $<5$ ng/ml?". Mol. Imaging. Biol., 8: 43, 2006.

28. KWEE, S.A.; WEI, H.; SESTERHENN, I. y cols.: "Localization of primary prostate cancer with dual-phase 18F-Fluorocholine PET". J. Nucl. Med., 47: 262, 2006.
*29. EVEN-SAPIR, E.; METSER, U.; MISHANI, E. y cols.: "The detection of bone metastases in patien-ts with high-risk prostate cancer: $99 \mathrm{mTc}-$ MDP planar bone scintigraphy, single- and multi-field of view SPECT, 18F-fluoride PET and 18F-fluoride PET/CT”. J. Nucl. Med., 47: 287, 2006.

30. TOTH, G.; LEBGYEL, Z.; BALKAY, L. y cols.: "Detection of prostate cancer with 11C-methionine positron emission tomography”. J. Urol., 173: 66, 2005.

31. ZANZONICO, P.B.; FINN, R.; PENTLOW, K.S. y cols.: "PET-based radiation dosimetry in man of 18F-fluorodihydrotestoterone, a new radiotracer for imaging prostate cancer". J. Nucl. Med., 45: 1966, 2004.

32. LARSON, S.M.; MORRIS, M.; GUNTHER, I. y cols.: "Tumor localization of $16 \mathrm{~b}(18)$ F-fluoro-5adihydrotestosterone versus (18)F-FDG in patients with progressive, metastatic prostate cancer". J. Nucl. Med., 45: 366, 2004.

33. DESHDASHTI, F.; PICUS, J.; MICHALSKI, J.M. y cols.: "Positron tomographic assessment of androgen receptors in prostatic carcinoma". Eur. J. Nucl. Med. Mol. Imaging., 32: 344, 2005.

34. JACOBSON, O.; BECHOR, Y.; ICAR, A. y cols.: "Prostate cancer PET bioprobes: síntesis of 18F-radiolabeled hydroxyflutamide derivates". Bioorg. Med. Chem., 13: 6195, 2005.

35. FOSS, C.A.; MEASE, R.C.; FAN, H. y cols.: "Radiolabeled small-molecule ligands for prostate-specific membrane antigen: in vivo imaging in experimental models of prostate cancer". Clin. Cancer Res., 11: 4022, 2005.

36. ZHANG, X.; CAI, W.; CAO, F. y cols.: "18F-labeled bombesin analogs for targeting GRP receptor -expressing prostate cancer". J. Nucl. Med., 47: 492, 2006.

37. MULLERAD, M.; EISENBERG, D.P.; AKHURST, T. y cols.: "Use of positron emission tomography to target prostate cancer gene therapy by oncolytic herpes simple virus". Mol. Imaging. Biol., 8: 30, 2006.

38. JOHNSON, M.; SATO, M.; BURTON, J. y cols: "Micro-PET/CT monitoring of herpes thymidione kinase suicide gene therapy in a prostate cancer xenograft: the advantage of a cell-specific transcriptional targeting approach". Mol. Imaging., 4: 463, 2005.

39. SATO, M.; JOHNSON, M.; ZHANG, L. y cols: "Functionality of androgen receptor-based gene expression imaging in hormone refractory prostate cancer". Clin. Cancer Res., 11: 3743, 2005.

40. ALESSIO, A.; KINAHAN, P.E.; CHENG, P.M. y cols.: "PET/CT scanner instrumentation, challenges, and solutions". Radiol. Clin. N. Am., 42: 1017, 2004. 